\title{
CDISC SDTM Treatment Episode Terminology
}

National Cancer Institute

\section{Source}

National Cancer Institute. CDISC SDTM Treatment Episode Terminology. NCI

Thesaurus. Code C102589.

Terminology associated with the treatment episode codelist of the Clinical Data

Interchange Standards Consortium (CDISC) Study Data T abulation Model (SDT M). 